\title{
Education and Consumer Informatics: Improvements in Existing Systems
}

\section{Findings from the Yearbook 2008 Section on Education and Consumer Informatics}

\author{
C. Boyer, Managing Editor for the IMIA Yearbook Section on Education and Consumer Informatics \\ Health On the Net Foundation (HON), Geneva, Switzerland
}

\section{Introduction}

The need for patient health education through vehicles other than the health professional is growing day by day. This is especially felt now, in a time which is fast paced, oriented towards efficiency. As discussed in the paper presented in the Education and Consumer Health Informatics sector of the IMIA Yearbook 2007, Marschollek [1] speaks of the need for varying methods of communication between laypersons and professionals, especially so in the domain of health and healthcare. He presented four papers, of which three describe systems used for improving the communication between health professionals and patients. While various tools and systems are required to improve the means by which patients obtain health information, it is also important to gauge their ability to understand and then implement this information successfully. In addition, it should always be remembered that technology is not a means of replacing, but rather an augmentation of the health professional.

\section{Best Paper Selection}

The selected best papers for the section Education and Consumer Health Informatics 2008 describe and address the various issues mentioned above. Two of the five papers selected, describe innovative systems to help improve the existing communications between health professionals and patients as well as those which provide patients with new ways of obtaining health information. Valenzuela et al. present a teleconsultation system mainly targeted towards the rural populations to improve health education and act as a screening or triage tool for consultations in Columbia. The difficulties to be overcome for proper implementation are also described. Norman et al. present a simple yet functional tool to measure the eHealth literacy of consumers concluding that it will enable identifying of comprehension gaps and assistance of those with lower comfort levels to benefit from the advantages of eHealth. Sari et al. show in their study the possible gaps in a routine reporting system for patient safety incidents in a hospital and suggest that augmentation of this system with routine case note review could be used to provide better screening thus giving a better reflection of the actual situation. Several other studies have also shown that there is a considerable amount of citizen who use the Internet health services on a regular basis, and these usually share various common demographic and health factors. For example, [7] shows that the majority 
Table 1 Best paper selection of articles for the IMIA Yearbook of Medical Informatics 2008 in the section 'Education and Consumer Informatics'. The articles are listed in alphabetical order of the first author's surname.

Section

Education and Consumer Informatics

- Andreassen HK, Bujnowska-Fedak M M, Chronaki CE, Dumitru RC, Pudule I, Santana S, Voss H, Wynn R. European citizens' use of E-health services: A study of seven countries. BMC Public Health 2007; 7(147): 53

- Hurling R, Catt M, De Boni M, Fairley BW, Hurst T, Murray P, Richardson A, Sodhi JS. Using Internet and Mobile Phone Technology to Deliver an Automated Physical Activity Program: Randomized Controlled Trial. J Med Internet Res 2007; 9 (2): e7

- Norman CD, Skinner HA. eHEALS : The eHealth Literacy Scale. J Med Internet Res. 2006;8(4):e27

- Sari ABA, Sheldon TA, Cracknell A, Turnbull A. Sensitivity of routine system for reporting patient safety incidents in an NHS hospital: retrospective patient case note review. BMJ 2007 Jan 13, 334(7584):79.

- Valenzuela JI, Arguello A, Cendales JG, Rizo CA. Web-Based Asynchronous Teleconsulting for Consumers in Colombia: A Case Study. J Med Internet Res 2007; 9(4):e33

- Zeng QT, Tse T, Divita G, Keselman A, Crowell J, Browne AC, Goryachev S, Ngo L. Term Identification Methods for Consumer Health Vocabulary Development. J Med Internet Res 2007;9(1):e4

of Internet users for health purposes are women of middle age with a good education. Andreassen et al. demonstrate a similar picture to the one described above in their study. Hurling et al. show the impact of Internet and mobile phone based health programs on the behaviour of the public.

\section{Conclusions and Outlook}

The selection of papers reflect that, although patient education and health informatics have been well integrated and resulted in a growing population of informed consumers, there are still barriers to be overcome and gaps to be filled, if we are to reach ever wider communities. This can be done with valuable and simple tools such as those described above and although each has its own barriers, there is room for further development and improvement from these foundations. The possibilities of patient health education through the web are immense, with the one limitation that it will never surpass the patient-physician bond. Keeping this in mind, the web can be harnessed as one of the key tools for health and healthcare.

\section{References}

1. Marschollek M. Advances in Education and Consumer Health Informatics: Findings from the section on Education and Consumer Informatics Methods.Inf Med 2007;46 Suppl 1: 95-7.

2. Valenzuela JI, Arguello A, Cendales JG, Rizo CA. Web-based Asynchronous Teleconsulting for Consumers in Colombia: A Case Study. J Med Internet Res 2007;9(4):e33.

3. Norman CD, Skinner HA. eHEALS: The eHealth Literacy Scale. J Med Internet Res 2006;8(4):e27.

4. Sari ABA, Sheldon TA, Cracknell A, Turnbull A. Sensitivity of routine system for reporting patient safety incidents in an NHS hospital: retrospective patient case note review. BMJ 2007 Jan 13; 334(7584):79.

5. Andreassen HK, Bujnowska-Fedak MM, Chronaki CE, Dumitru RC, Pudule I, Santana S, Voss H, Wynn R. European citizens' use of E-health services: A study of seven countries. BMC Public Health 2007,7:53.

6. Hurling R, Catt M, De Boni M, Fairley BW, Hurst T, Murray P, Richardson A, Sodhi JS. Using Internet and Mobile Phone Technology to Deliver an Automated Physical Activity Program: Randomized Controlled Trial. J Med Internet Res 2007; 9(2):e7

7. Renahy E, Parizot I, Lesieur I, Chauvin P. WHIST. Enquete web sur les habitudes de $\mathrm{r}$ e $\mathrm{c}$ h e $\mathrm{r} \mathrm{c}$ h e d'informations liées à la santé sur Internet. http:// www.u707.jussieu.fr/ds3/, last accessed on March 18, 2008.

\section{Correspondence to:}

Celia Boyer

Health On the Net Foundation (HON)

c/o Geneva University Hospitals / SIM

24 rue Micheli-du-crest

1211 Geneva 14

Switzerland

E-mail: celia.boyer@healthonnet.org
Appendix: Content Summaries of Selected Best Papers for the IMIA Yearbook 2008, Section Education and Consumer Informatics*

\author{
Valenzuela J,,ArguelloA, CendalesJG, Rizo CA \\ Web-Based Asynchronous Teleconsulting for \\ Consumers in Colombia: A Case Study
}

\section{J Med Internet Res 2007;9(4):e33}

This paper describes the experience of the Centre for Virtual Education and Simulation eHealth in Columbia with open-access web-based tele-consultation for consumers. The answers received from the Spanish tele-consultation tool were classified on 3 axes: purpose of query; speciality and geographic area of query. The authors report that more females than males used the tool during a period of 6 months, the majority of all users being aged 24 to 29 . The largest proportion of answers were received from Columbia with other Spanish speaking countries like Spain, Mexico, Argentina and others including the United States forming a minority group. However, most of the responses were received from the capital and 4 other main cities of $\mathrm{Co}$ lumbia while only 2 out of 270 requests came from rural areas of the country and 40 requests were from intermediate cities. This was thought to be partly due to the limited access to the Web in rural areas. The authors conclude that tele-consultation could be a useful tool in providing health education, acting as a triage system for those waiting for face to face consultations and overall empower consumers. Barriers to overcome however are cultural, infrastructural and connectivity issues before it can be successfully implemented for all communities.

* The complete papers can be accessed in the Yearbook's full electronic version, provided that permission has been granted by the copyright holder(s) 


\section{Norman CD, Skinner HA}

\section{eHEALS : The eHealth Literacy Scale}

\section{J Med Internet Res. 2006; vol. 8; iss. 4; e27}

This paper reports on an evaluation of the eHEALS system in measuring the consumers' combined knowledge, comfort and skills in finding, evaluating and applying eHealth information to health problems. The eHEALS is an 8-item measure with a 5-point Likert scale. The six core skills of eHealth literacy, namely, traditional literacy, health literacy, scientific literacy, media literacy, information literacy, and computer literacy were measured from a group of youth aged 13 to 21 of multiple ethnicities and from varying backgrounds, at baseline, post-intervention, 3 month, and 6 month intervals. The authors report on a baseline rise of eHealth literacy in males as compared to females but no statistical significance at post-intervention, 3 month or 6 month follow-up. There was no significant relationship between eHealth literacy and information technology and knowledge of any other technology like mobile phones, email or pagers. eHealth literacy was also not significantly related to prediction of perceived health status over time. They conclude that the simplicity of eHEALS makes it a probable standard assessment tool for gauging eHealth literacy in health care, allowing identification of skill gaps and assistance for relevant persons.

\section{Sari ABA, Sheldon TA, Cracknell A, Turnbull A Sensitivity of Routine System for Reporting Patient Safety Incidents in an NHS Hospital: Retrospective Patient Case Note Review BMJ 2007 Jan 13;334(7584):79.}

Routine hospital reporting of patient safety incidents, despite promising tools in place, is sometimes inadequate and may not project the true picture. The authors describe an evaluation of the performance of a routine safety incident reporting system in a UK hospital. A random sample of 1006 admissions from 8 specialities were selected and the data was reviewed in two ways, through a 2-stage retrospective case note review, and through a review of the patient safety incidents reported by the routine hospital reporting system. Stage one of the case note review was carried out by five nurses who screened records looking for 18 pre-described criteria. One or more positive criteria were considered as indicators for patient safety incidents. In stage 2 , three doctors reviewed all the positive records using a structured review form to judge the actual occurrence of a patient safety incident and if so, it's type and consequences. The conclusion of this study was that the routine hospital reporting system for patient safety incidents may not provide an accurate picture of the actual number and severity of patient safety incidents occurring and routine structured case note review on samples of medical records should be considered for quality improvement.

\section{Andreassen HK, Bujnowska-Fedak M M, Chronaki CE, Dumitru RC, Pudule I, Santana S, Voss H, Wynn R \\ European Citizens' Use of E-health Services: A Study of Seven Countries \\ BMC Public Health. 2007 Apr 10;7(147):53}

The Internet is increasingly being utilized in health care, and many medical professionals encourage their patients to use Internet health services. The study described was performed on a sample of the general population in 7 European countries, namely, Norway, Denmark, Germany, Greece, Poland, Portugal and Latvia. The total sample consisted of 7934 respondents and interviews were conducted via telephone. Results showed that $71 \%$ of the Internet users, $44 \%$ of the total sample, had used the Internet for health purposes. Positive factors for Internet use for health purposes were youth, higher education, white-collar or no paid jobs, visits to the GP during the past year, long term illness or disability and a subjective assessment of one's health as being good. More women than men used the Internet for health purposes and the feeling of being reassured after use was twice as common as the feeling of being anxious. The conclusion of this study is that the users of Internet health services differ from the general public in demographic and health variables. The most common use of Internet health services is for information, followed by decision making regarding seeing a doctor and preparation and follow-up of a doctor's appointment. It was also seen that health-related use of the Internet does affect patients' use of other health services but is a supplement and not a replication of other health services.

\section{Hurling R, Catt M, De Boni M, Fairley BW, Hurst T, Murray P, Richardson A, Sodhi JS Using Internet and Mobile Phone Technol- ogy to Deliver an Automated Physical Activ- ity Program: Randomized Controlled Trial}

\section{J Med Internet Res 2007;9 (2):e7}

The Internet has the potential of being a major medium for promoting health behaviour changes. However no controlled studies have been performed to evaluate to what extent it can help. The authors describe a randomized controlled trial done to evaluate the impact of a 9week physical activity program based on the Internet and mobile phone technology in Bedfordshire in the United Kingdom. 77 adults of and average age of 40 years and an average BMI of 26.3 were randomized into test group and control group and both groups were provided with a wrist accelerometer to monitor their level of physical activity. Only the test group received feedback via an Internet and mobile phone based physical activity program in the test group while the control group received no support. The results showed a significant increase in intention and expectation to exercise in the test group than the control group and the test group had lost more body fat than the control group. The conclusion of this study is that a fully automated Internet and mobile phone based motivation and action support system can significantly increase and maintain the level of physical activity in adults. 


\section{Zeng QT, Tse T, Divita G, Keselman A,} Crowell J, Browne AC, Goryachev S, Ngo L Term Identification Methods for Consumer Health Vocabulary Development*

\section{J Med Internet Res. 2007; 9(1):e4}

This paper describes the research performed on developing an online consumer health vocabulary (CHV) prompted by the development of numerous online consumer health information applications. Valid terms (term hood determination) were picked from 1893 identified candidate strings (words/phrases) through several methods including collaborative human review and automated term recognition methods (C-value formula and logistic regression model). The authors report a higher distinguishing power from the logistic regression model with $95.5 \%$ (where $100 \%$ is perfect discriminative ability, $50 \%$ is no ability, $<50 \%$ is incorrect predictions) as compared to the C-value method with $70.9 \%$. They conclude that both collaborative human review and logistic regression methods were effective for identifying terms for CHV development.

* The complete paper can be accessed in the Yearbook's full electronic version, provided that permission has been granted by the copyright holder(s) 